\title{
RECURRENCE FORMULAS FOR CERTAIN DIVISOR FUNCTIONS
}

\author{
D. H. LEHMER
}

Numerical functions giving the excess of the number of divisors of $n$ of one sort over the number of divisors of a second sort were introduced over a century ago from the theory of binary quadratic forms and from the theory of elliptic functions. Later a systematic discussion of several of these functions was made by J. W. L. Glaisher, who, by means of theta function identities, found recurrences with gaps for these functions. The purpose of this note is to point out that these functions are sums over divisors of certain periodic Lucas functions and that sums of Lucas functions in general satisfy triangular number recurrence relations as given by (6) below. This formula is specialized in several ways, first to periodic Lucas functions, then to degenerate functions, and then to the more typical cases related to the Pell equation and Fibonacci's series.

Formula (6) may be obtained in the following elementary manner from the famous triple product identity of Gauss and Jacobi. ${ }^{1}$

$$
\prod_{\nu=1}^{\infty}\left\{\left(1-x^{2 \nu}\right)\left(1+a x^{2 \nu-1}\right)\left(1+a^{-1} x^{2 \nu-1}\right)\right\}=\sum_{n=-\infty}^{\infty} a^{n} x^{n} .
$$

If in (1) we set $x=t^{1 / 2}$ and $a=-\alpha t^{1 / 2}$ we obtain at once

(2) $(1-\alpha) \prod_{\nu=1}^{\infty}\left\{\left(1-t^{\nu}\right)\left(1-\alpha t^{\nu}\right)\left(1-\alpha^{-1} t^{\nu}\right)\right\}=\sum_{n=--\infty}^{\infty}(-\alpha)^{n+1} t^{n(n+1) / 2}$.

If now we introduce $\theta$ by $\alpha=e^{2 \theta i}$ and write

$$
P(\theta, t)=\prod_{\nu=1}^{\infty}\left\{\left(1-t^{\nu}\right)\left(1-2 t^{\nu} \cos 2 \theta+t^{2 \nu}\right)\right\},
$$

then, on combining the terms on the right of (2) for $n=r-1$ and $-r$, 1942.

Presented to the Society, September 10, 1942; received by the editors May 25,

${ }^{1}$ K. G. J. Jacobi, Fundamenta nova theoriae functionum ellipticarum, Königsberg, 1829, §64; Gesammelte Werke, Berlin, 1881, vol. 1 pp. 232-234. Other elementary proofs of (1) (not depending on elliptic functions) have been given by A. Cauchy, C. R. Acad. Sci. Paris vol. 17 (1843) pp. 523-531; Oeuvres, vol. 8, pp. 42-50; A. Enneper, Elliptische Functionen, Halle, 1876, pp. 74-77; J. Tannery and J. Molk, Éléments de la theorie des fonctions elliptiques, Paris, 1896, vol. 2 pp. 10-13; G. H. Hardy and E. M. Wright, An introduction to the theory of numbers, Oxford, 1938, pp. 280-281. 
we can write (2) in the form

$$
P(\theta, t)=\sum_{r=1}^{\infty} t^{r(r-1) / 2}(-1)^{r+1} \frac{\sin (2 r-1) \theta}{\sin \theta} .
$$

Differentiating (3) and (4) with respect to $\theta$ we find, on substituting for $P(\theta, t)$ from (4):

$$
\begin{aligned}
& \left\{\sum_{k=1}^{\infty}(-1)^{k} t^{k(k-1) / 2} \frac{\sin (2 k-1) \theta}{\sin \theta}\right\}\left\{\sum_{\nu=1}^{\infty} \frac{t^{\nu}}{1-2 t^{\nu} \cos 2 \theta+t^{2 \nu}}\right\} \\
& =(1 / 4) \csc ^{2} \theta \sum_{k=1}^{\infty}(-1)^{k} t^{k(k-1) / 2}\left\{\frac{k \cos (2 k-1) \theta}{\cos \theta}-\frac{\sin 2 k \theta}{\sin 2 \theta}\right\} .
\end{aligned}
$$

Let us consider the general Lucas function

$$
U_{n}=\frac{a^{n}-b^{n}}{a-b}
$$

where $a, b$ are the roots of $x^{2}-P x+Q=0$. Supposing that $Q \neq 0$ we define $q$ by

$$
q=Q^{-1 / 2}
$$

where a definite square root of $Q$ is taken. Finally we introduce the divisor sum $D_{n}$ by

$$
D_{n}=\sum_{\delta \mid n} U_{\delta} q^{\delta}
$$

where $\delta$ ranges over the positive divisors of $n$. Let the parameter $\theta$ in (5) be taken so that $2 \cos 2 \theta=P q$, and hence

$$
\frac{\sin 2 n \theta}{\sin 2 \theta}=q^{n-1} U_{n} \text {. }
$$

Then, since

$$
U_{n}=P U_{n-1}-Q U_{n-2},
$$

we have

$$
\begin{aligned}
U_{n} q^{n} & =P q U_{n-1} q^{n-1}-q^{2} Q U_{n-2} q^{n-2} \\
& =2 \cos 2 \theta U_{n-1} q^{n-1}-U_{n-2} q^{n-2} .
\end{aligned}
$$

Therefore

$$
\left(1-2 x \cos 2 \theta+x^{2}\right) \sum_{m=1}^{\infty} x^{m} q^{m} U_{m}=x q .
$$

Setting $x=t^{\nu}$ and summing over $\nu=1,2,3, \cdots$, we get 


$$
q \sum_{\nu=1}^{\infty} \frac{t^{\nu}}{1-2 t^{\nu} \cos 2 \theta+t^{2 \nu}}=\sum_{m=1}^{\infty} \sum_{\nu=1}^{\infty} t^{\nu m} q^{m} U_{m}=\sum_{n=1}^{\infty} D_{n} t^{n} .
$$

Substituting this into (5) and comparing coefficients of $t^{n}$ on both sides of the resulting equation we find

$$
\begin{gathered}
D_{n}-D_{n-1} \frac{\sin 3 \theta}{\sin \theta}+D_{n-3} \frac{\sin 5 \theta}{\sin \theta}-D_{n-6} \frac{\sin 7 \theta}{\sin \theta}+\cdots \\
=\left\{\begin{array}{cc}
(q / 4) \csc ^{2} \theta(-1)^{k+1}\left\{\frac{k \cos (2 k-1) \theta}{\cos \theta}-\frac{\sin 2 k \theta}{\sin 2 \theta}\right\} \\
0 & \text { if } n=k(k-1) / 2 \\
\text { otherwise. }
\end{array}\right.
\end{gathered}
$$

Here the left side involves only such $D$ 's as have subscripts differing from $n$ by triangular numbers.

Of the special cases of (6) perhaps the most interesting are those in which $q=1$ and $\theta$ is a simple rational multiple of $\pi$. In these cases the function $D_{n}$ belongs to a general class which is composed of functions of which the typical one may be denoted by

$$
E_{m}(n)=E_{m}\left(n \mid \alpha_{1}, \alpha_{2}, \cdots ; \beta_{1}, \beta_{2}, \cdots\right)
$$

and is defined as the excess of the number of those divisors of $n$ of one of the forms $m x+\alpha_{i}$ over the number of those divisors of $n$ of one of the forms $m x+\beta_{i}$.

Thus for $q=1, \theta=\pi / 4$ we have

$$
U_{n}=\frac{\sin n \pi / 2}{\sin \pi / 2}=\left\{\begin{array}{rll}
+1 & \text { if } & n=4 x+1 \\
-1 & \text { if } n=4 x+3 \\
0 & \text { otherwise. }
\end{array}\right.
$$

Hence

$$
D_{n}=\sum_{\delta \mid n} U_{\delta}=E_{4}(n)=E_{4}(n \mid 1 ; 3) .
$$

In this case (6) gives us

$$
\begin{gathered}
E_{4}(n)-E_{4}(n-1)-E_{4}(n-3)+E_{4}(n-6)+E_{4}(n-10)-\cdots \\
=\left\{\begin{array}{cl}
(-1)^{[(k-1) / 2][k / 2]} & \text { if } n=k(k-1) / 2 \\
0 & \text { otherwise. }
\end{array}\right.
\end{gathered}
$$

This formula is due to Glaisher. ${ }^{2}$

${ }^{2} \mathrm{~J}$. W. L. Glaisher, On the function which denotes the difference between the number of $4 m+1$-divisors and the number of $4 m+3$-divisors of a number, Proc. London Math. Soc. (1) vol. 15 (1884) pp. 104-122 (especially pp. 107, 110). 
If $\theta=\pi / 3$ we find that $U_{n}=0,1$ or -1 according as $n$ is congruent to 0,1 , or 2 modulo 3 . Hence

$$
D_{n}=E_{3}(n)=E_{3}(n \mid 1 ; 2) .
$$

In this case (6) gives

$$
\begin{gathered}
E_{3}(n)-E_{3}(n-3)-E_{3}(n-6)+E_{3}(n-15)+E_{3}(n-21)-\cdots \\
=\left\{\begin{array}{cl}
(2 k-1) / 3 & \text { if } n=k(k-1) / 2 \text { and } k \equiv 2(\bmod 3) \\
-[(k+1) / 3] & \text { if } n=k(k-1) / 2 \text { and } k \neq 2(\bmod 3) \\
0 & \text { otherwise. }
\end{array}\right.
\end{gathered}
$$

Here the numbers $0,3,6,15,21,36,45, \cdots$ are those triangular numbers which are multiples of 3 . This formula, for the case $n \equiv 1$ $(\bmod 3)$, was given by Glaisher. ${ }^{3}$ If $\theta=\pi / 6$ then

$$
D_{n}=E_{6}(n)=E_{6}(n \mid 1,2 ; 4,5) .
$$

The recurrence (6) gives, in this case,

$$
\begin{gathered}
E_{6}(n)-2 E_{6}(n-1)+E_{6}(n-3)+E_{6}(n-6)-2 E_{6}(n-10) \\
+E_{6}(n-15)+E_{6}(n-21)-\cdots
\end{gathered}
$$

Here the coefficient of $E_{6}(n-\Delta)$ is +1 or -2 according as $\Delta$ is or is not a multiple of 3 .

In case $\theta$ is taken as $\pi / 8$, the function $D_{n}$ is no longer an integer, for all $n$, but has the value

$$
D_{n}=E_{8}(n \mid 1,3 ; 5,7)+2^{1 / 2} E_{8}(n \mid 2 ; 6) .
$$

The equation (6) now yields two formulas obtained by equating separately the rational and irrational parts of both sides. Combining these two results for simplicity we obtain the following, where

$$
E_{8}(n)=E_{8}(n \mid 1,3 ; 5,7) \text {. }
$$

If $n$ is even

${ }^{3} \mathrm{~J}$. W. L. Glaisher, On the function wich denotes the excess of the number of divisors of a number which $\equiv 1, \bmod 3$, over the number which $\equiv 2$, mod 3, Proc. London Math. Soc. (1) vol. 21 (1890) pp. 395-402. 


$$
\begin{gathered}
E_{8}(n)-E_{8}(n-1)+E_{8}(n-3)-E_{8}(n-6)-E_{8}(n-10)+\cdots \\
=\left\{\begin{array}{cl}
4[k / 4](-1)^{k+1+[k / 4]} & \text { if } n=k(k-1) / 2 \\
0 & \text { otherwise. }
\end{array}\right.
\end{gathered}
$$

If $n$ is odd

$$
\begin{aligned}
& E_{8}(n)+E_{8}(n-1)-E_{8}(n-3)-E_{8}(n-6)-E_{8}(n-10)-\cdots \\
& =\left\{\begin{array}{cc}
(-1)^{[k / 4]}\left\{6[(k+1) / 4]-[k / 2]+(-1)^{k} 2\right\} & \text { if } n=k(k-1) / 2 \\
0 & \text { otherwise. }
\end{array}\right.
\end{aligned}
$$

In the first of these the periodic sign pattern is

$$
+-+--+-\dot{t}+-+-+\cdots
$$

while in the second formula it is

$$
++---+\dot{+}++---+\dot{+} \cdots \text {. }
$$

As a by-product we have for $n$ odd:

$$
\begin{gathered}
E_{8}^{\prime}(n-1)-E_{8}^{\prime}(n-3)-E_{8}^{\prime}(n-15)+E_{8}^{\prime}(n-21) \\
\quad+E_{8}^{\prime}(n-45)-E_{8}^{\prime}(n-55)-\ldots \\
=\left\{\begin{array}{cl}
(-1)^{[k / 4]} 2[(k+1) / 4] & \text { if } n=k(k-1) / 2 \\
0 & \text { otherwise. }
\end{array}\right.
\end{gathered}
$$

Here we have

$$
E_{8}^{\prime}(n)=E_{8}(n \mid 1,2,3 ; 5,6,7) .
$$

The numbers $1,3,15,21,45, \cdots$ are the odd triangular numbers and the sign pattern is

$$
+--++--++-++-+\cdots \text {. }
$$

The values 0 and $\pi / 2$ of $\theta$ correspond to degenerate values of Lucas $U_{n}$ which becomes $n$ and $(-1)^{n+1} n$, respectively. If we take the limiting forms of (6) in these cases we get the following two formulas

$$
\begin{aligned}
& \begin{aligned}
& \sigma(n)-3 \sigma(n-1)+5 \sigma(n-3)-7 \sigma(n-6)+9 \sigma(n-10)-11 \sigma(n-15)+\cdots \\
&=\left\{\begin{array}{cl}
(-1)^{k} k(k-1)(2 k-1) / 6 & \text { if } n=k(k-1) / 2 \\
0 & \text { otherwise; }
\end{array}\right. \\
& \lambda(n)+\lambda(n-1)+\lambda(n-3)+\lambda(n-6)+\lambda(n-10)+\ldots
\end{aligned} \\
& = \begin{cases}n & \text { if } n \text { is triangular } \\
0 & \text { otherwise. }\end{cases}
\end{aligned}
$$

In the first formula $\sigma(n)$ denotes the sum of the divisors of $n$ and in 
the second $\lambda(n)$ denotes the excess of the sum of the odd divisors of $n$ over the sum of its even divisors. The first relation, apparently, was first published by $\mathrm{Sardi}^{4}$ and later given by Halphen ${ }^{5}$ and Glaisher ${ }^{6,7}$ The second is due to Glaisher ${ }^{1}$ and Lipschitz. ${ }^{8}$

Two final examples of (6) in which $\theta$ is complex will be given. The first of these corresponds to $q=1$ and $\theta=\arcsin i$. Let $\left(x_{m}, y_{m}\right)$ be the $m$ th multiple solution of the Pell equation

$$
x^{2}-2 y^{2}=1
$$

and $\left(t_{m}, u_{m}\right)$ be the $m$ th solution of the modified Pell equation

$$
t^{2}-2 u^{2}=-1
$$

so that

$$
\begin{aligned}
& y_{1}=2, y_{2}=12, y_{3}=70, \cdots, y_{i+1}=6 y_{i}-y_{i-1}, \\
& t_{1}=1, t_{2}=7, t_{3}=41, \cdots, t_{i+1}=6 t_{i}-t_{i-1}, \\
& u_{1}=1, u_{2}=5, u_{3}=29, \cdots, u_{i+1}=6 u_{i}-u_{i-1} .
\end{aligned}
$$

Finally let

$$
S(n)=\sum_{\delta \mid n} y_{\delta}
$$

Then (6) becomes

$$
\begin{gathered}
t_{1} S(n)-t_{2} S(n-1)+t_{3} S(n-3)-t_{4} S(n-6)+t_{5} S(n-10)-\cdots \\
=\left\{\begin{array}{cl}
(-1)^{k}\left(2 k u_{k}-y_{k}\right) / 4 & \text { if } n=k(k-1) / 2 \\
0 & \text { otherwise. }
\end{array}\right.
\end{gathered}
$$

The case of $\theta=\operatorname{arc} \sin (i / 2)$ has to do with the Fibonacci series:

$$
F_{1}=1, F_{2}=1, F_{3}=2, F_{4}=3, F_{5}=5, \cdots, F_{i+1}=F_{i}+F_{i-1} \text {. }
$$

${ }^{4}$ C. Sardi, Sulle somme dei divisori dei numeri, Giornale di Mathematica vol. 7 (1869) pp. 112-115.

${ }^{5} \mathrm{G}$. Halphen, Sur une formule récurrente concernant les sommes des diviseurs des nombres entiers, Bull. Soc. Math. France vol. 5 (1877) pp. 158-160.

${ }^{6} \mathrm{~J}$. W. L. Glaisher, Theorems relating to the sum of the uneven divisors of a number, Quart. J. Math. vol. 19 (1883) pp. 216-223 (especially p. 220).

${ }^{7} \mathrm{~J}$. W. L. Glaisher, On the sum of the divisors of a number, Proc. Cambridge Philos. Soc. vol. 5 (1884), pp. 108-120.

8 R. Lipschitz, Sur les sommes des diviseurs des nombres, C. R. Acad. Sci. Paris vol. 100 (1885) pp. 845-847. (The recurrence (7), which is misquoted in Dickson's History of the theory of numbers vol. 1, p. 302, was given without proof six years earlier by Glaisher, Nouvelle Correspondance Mathématique vol. 5 (1879) p. 176.) 
In fact if we let

$$
T(n)=\sum_{\delta \mid n} F_{2 \delta}
$$

then

$$
\begin{aligned}
& T(n)-4 T(n-1)+11 T(n-3)-29 T(n-6)+\cdots \\
&=\left\{\begin{array}{cl}
(-1)^{k} k F_{2 k-1}-F_{2 k} & \text { if } n=k(k-1) / 2 \\
0 & \text { otherwise. }
\end{array}\right.
\end{aligned}
$$

Here the $m$ th term of the sequence

$$
1,4,11,29,76,199, \ldots
$$

is

$$
F_{2 m}+F_{2 m-2}
$$

UNIVERSITY OF CALIFORNIA

\section{ON PARTICULAR SOLUTIONS OF LINEAR PARTIAL DIFFERENTIAL EQUATIONS}

\section{K. L. NIELSEN AND B. P. RAMSAY}

1. Introduction. The boundary value and characteristic value problems are classical questions in the theory of partial differential equations of elliptic type. A method for actual solution of these problems consisting of approximations by expressions $W_{n}=\sum_{\nu=1}^{n} \alpha_{\nu}^{(n)} \phi_{\nu}(x, y)$, where $\phi_{\nu}(x, y)$ are particular solutions of the considered differential equation, has been given by Bergman (see [1]). ${ }^{1}$ Here the $\alpha_{\nu}^{(n)}$ are constants which are to be determined by the requirement that the values of $W_{n}$ on the boundary approximate the given data (for details see [1]). ${ }^{2}$

In applying this method it is important for practical purposes to obtain a simple procedure for the construction of the particular solu-

Presented to the Society, September 10,1942; received by the editors May 13, 1942. This paper was prepared while the authors were fellows under the program of Advanced Instruction and Research in Mechanics at Brown University.

1 The numbers in the brackets refer to the bibliography.

${ }^{2}$ This method is in a certain sense the reverse of the Rayleigh-Ritz method in which the approximating expressions satisfy the boundary conditions but do not satisfy the given equation. 